\title{
An Interconnected Smart Technology System for Individuals with Mental Illness Living in the Community and Transitional Hospital Apartments
}

\author{
Cheryl Forchuk ${ }^{1,2(\bowtie)}$, Jonathan Serrato ${ }^{1}$, Abraham Rudnick ${ }^{3}$, \\ Deborah Corring $^{2}$, Rupinder Mann ${ }^{1}$, and Barbara Frampton ${ }^{4}$ \\ ${ }^{1}$ Lawson Health Research Institute, London, ON, Canada \\ cforchuk@uwo.ca \\ 2 Western University, London, ON, Canada \\ 3 Dalhousie University, Halifax, NS, Canada \\ ${ }^{4}$ CONNECT for Mental Health, London, ON, Canada
}

\begin{abstract}
The overall objective of this research was to develop and test the use of smart technology in delivering safe, effective mental health services before expanding into community homes. A system was created that linked multiple screen devices such as smartphones and tablets and health monitoring devices with a central secure database for data to be funneled and stored for monitoring and tracking. In order to assess the feasibility of this technological innovation, the research team installed equipment in two prototype apartments at two inpatient psychiatric hospitals and in up to eight community homes operated by the Canadian Mental Health Association and London Middlesex Community Housing. The results indicate that most participants found the technology acceptable, and that the system was successfully able to export data securely.
\end{abstract}

Keywords: Smart technology $\cdot$ Mental illness $\cdot$ Health management $\cdot$ Housing $\cdot$ Data management

\section{Introduction}

In Canada, the Mental Health Commission of Canada estimates that $20 \%$ of the population will experience a mental illness during their lifetime while the remaining $80 \%$ will be affected by the mental illness of others [1]. Mood and psychotic disorders have also been linked with polydipsia [2] and cardiovascular disease [3]. It is therefore important for comorbidities to be observed in order to understand the complete health status of the individual. This is of particular importance given the patient-specific issues in Canada such as travel-related barrier for accessing community mental health care as well as long wait-times, lack of information, and stigma [4]. Furthermore, previous research has revealed increased mortality rates among individuals with severe mental illness due to a lack of self-management for acute and chronic illnesses [5]. Impairment in activities of daily living, function (e.g. inability to maintain a healthy diet, lifestyle, etc.) and cognition can result in missed medications, appointments, or self-care activities. Individuals 
with these types of impairments may not realize their self-care is declining therefore causing greater declines in mental and physical health. Symptom tracking and monitoring, enhanced awareness of pre-symptoms, and greater access to informational resources through smart technology may also aid patient well-being and beneficence [6].

In previous studies conducted by this research team, correlations between mental health and housing have been demonstrated but housing alone is not enough to promote stabilization [7]. While mental illness is often conceptualized as a problem between the individual and their environment, mental health care is almost entirely focused on "fixing" the individual rather than the environment they are in. To that end, previous research has demonstrated promising findings from the implementation of mobile and web-based technologies in a population of individuals experiencing mood or psychotic disorders [8-11]. Forchuk et al. revealed a $48.6 \%$ reduction in hospitalizations and $57 \%$ fewer outpatient visits after 18 months using mobile phones for prompts and reminders along with access to a personal health record [12]. This current project therefore reflects a logical extension of these studies by developing a "high-dose" smart technology intervention for those with severe mental illnesses; first by starting in hospital prototype apartments before extending further into community homes. Two reviews have revealed that there is a lack of evaluation of smart technology within a health care context $[13,14]$. One of which, a Cochrane review, revealed no health-related smart technology studies that met the Cochrane Handbook criteria for inclusion and none of the identified studies evaluated effectiveness [14].

The groundwork of this project, laid by Corring, Campbell and Rudnick [8], aims to provide supportive systems within an individual's environment, be it within one's home or a hospital transitional apartment to promote community integration, to facilitate chronic illness management, and support independence. Furthermore, this project will attempt to establish the use of smart technology in assisting individuals with mental illness and cognitive impairment in a hospital apartment setting first before progressing and expanding into community homes. The objective of these phases was to develop and test smart technology in delivering safe, effective mental health services. As such, we hypothesize that this smart technology intervention will result in:

1. An increase in participants' level of community integration.

2. An increase in participants' housing stability.

3. A decrease in excessive health and social service utilisation.

4. An improvement in the participants' overall health.

At present, the hospital prototype phase has been ongoing for approximately 7 months. The community phase has seen equipment installed in five homes and is continuing to enroll participants.

\section{Materials and Methods}

\section{Design}

This quasi-experimental project is separated into two phases. The hospital prototype phase is employing a within-group, mixed-methods, descriptive pilot design in order to 
ascertain the feasibility of the smart technology in a hospital setting as well as testing the system as a whole. Data was collected upon discharge from the hospital apartment and at 6-month follow-up.

As the project moves from the hospital prototype phase, the community phase is adopting a more longitudinal approach in testing the project's hypotheses. This involves a within-group, mixed-methods, repeated-measures design. Data is being collected over three assessments conducted at baseline, 6-month and 12-month followups. Comparisons regarding levels of community integration, health, housing stability and service usage throughout the intervention will be observed. Ethical approval was obtained through Western University's Research Ethics Board and Lawson Health Research Institute.

\section{Description of the Settings}

Health Care Providers (HCPs) at two inpatient psychiatric facilities and HCPs in community homes coordinated with the research staff to set up various smart technologies in the apartments for individuals with mental illness. The prototype apartments within the psychiatric facilities are available to individuals who were being discharged into the community in order to provide a transitional experience. The community homes are operated by the Canadian Mental Health Association (CMHA) and London Middlesex Community Housing (LMCH). These include group homes, family homes and individual apartments with staff members providing in-home care and support on a need-to-need basis.

\section{The System}

This project has sought to incorporate real-time integration of data in order to provide HCPs with notifications and monitoring capabilities. The system links multiple smart technology devices and funnels the data into one database. This system has been comprised of two software innovations; the Lawson Integrated Database (LIDB) and the Collaborative Health Record (CHR).

The LIDB is an information management platform that collates and manages client health information behind the St. Joseph's Health Care hospital firewall. The LIDB keeps health data segregated in its own database schema but is capable of matching patient data across HCPs. With funds from the Canada Health Infoway, the research team was able to ensure the security of the LIDB through a third-party Privacy Impact Analysis and Threat Risk Assessment. Encrypted incremental data backups are performed on a nightly basis and full backups performed weekly with both stored securely off-site. The LIDB also utilizes virtual servers to move from one server to another to enable continuous operations with no impact to users. Weekly meetings with software engineers and monthly meetings with the hospital I.T. and Privacy departments have been held since the commencement of the project to address any data security concerns and ensure the integrity of the system. HCPs are able to log-in to the LIDB to view data from the health monitoring devices and the CHR, and also to set the reminders to be transmitted to the screen devices.

The CHR allows for both synchronous and asynchronous communication between patients and HCPs to deliver team-based, longitudinal health care. The CHR operates on the screen devices offered to the participants. The specific functions of the CHR include: 
1. Access to personal health information and self-assessments to enhance early identification of concerns related to symptoms.

2. A comprehensive patient-record system that provides workflows for a diverse group of HCPs.

3. Prompts and reminders that can support care planning for symptoms and comorbidities (e.g. medication reminders and activity prompts).

4. Secure communication between HCPs and participants including videoconferencing and messaging.

This functionality aids the complex care of people with severe mental illnesses by creating an enhanced secure connection between them and their circle of care. This helps overcome barriers to care such as mobility, transportation, or lack of resources readily available, and reduces the number of in-person appointments necessary. Self-

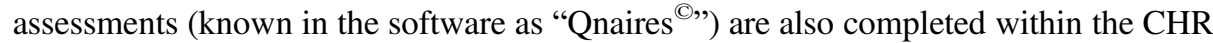
by the participant which allows the HCP and care team to monitor changes and potential crises. These self-assessments can include standardised tools used such as Patient Health Questionnaire (PHQ-9) and fully customisable tools created by the HCP within the CHR. The data from these assessments are then backed up to the participant's profile in the LIDB. In the event of a crisis (e.g. a participant indicates suicidal ideation on a Qnaire), an alert is sent to the care team so that they can act accordingly.

\section{Equipment}

Participants residing in the hospital prototype apartments can select a variety of screen devices including smartphones, tablets, and touch-screen monitors. These devices provide prompts and reminders generated by the LIDB to assist participants with cognitive deficits and facilitate self-care. The touch-screen monitors are developed inhouse by the research team's programmer. The monitor is programmed so that prompts and reminders on the screen can be "acknowledged" by the user by pushing the "Got It" button. This sends an automated message back to the HCP who set the reminder to inform them the reminder was received. Furthermore, a "Help" button was added after initial discussions with HCPs which sends a message to the participant's care team requesting them to provide support. This exhaustive approach of ensuring all devices were able to connect to each other and allowed for ease of data exporting to the LIDB was completed after weekly meetings and thorough testing.

In addition to the screen devices, the participants are offered a choice of adjunct health monitoring devices. In the hospital prototype phase, these include weigh scales, blood pressure monitors, glucometers, and a wearable activity tracker (smartwatch). These devices account for the comorbidities that may be present and will support chronic illness management. Data from these devices is pushed to the LIDB via encrypted authentication keys and SSL connectivity. The weigh scale exports data via WiFi whereas the glucometer, weigh scale and activity tracker utilize Bluetooth connectivity.

The screen devices on offer for individuals residing in the community homes differ slightly. Participants in this phase are offered smartphones and touch-screen monitors only. This was done to further refine the intervention and allow for an additional health adjunct health monitoring device to be offered. 
For participants in the community homes, their choice of health monitoring equipment also differs slightly with an automated medication dispenser being made available for selection but the blood pressure monitor and glucometer are not. The medication dispenser was not available for the hospital prototype phase due to medication protocols within the hospital. This addition of a medication dispenser represents the tailoring and refinement of the intervention for individuals living in the community. This was implemented to help participants with self-medication (if appropriate) and save time by reducing the need for participants and/or HCPs to collect medications from the pharmacy.

In both phases, participants are given the choice of devices they can use and are able to refuse any device with which they were not comfortable with. Participants recruited into the study are also allowed to refuse all devices but are still expected to complete a full semi-structured interview in order to acquire their opinions and attitudes as to why.

\section{Recruitment}

For all participants in both phases, the research team first recruited HCPs who then referred participants to the research team. For the hospital prototype phase of the project, the study is recruiting up to 20 participants and for the community phase, we are recruiting up to 13 participants. Participants are excluded from the study if they do not reside in the hospital prototype apartment for a minimum of one week.

Additional inclusion criteria for participants to participate in the community phase of the study include:

1. Must be on a caseload of a participating HCP.

2. Able to understand English to the degree necessary to participate.

3. Living in, eligible for, and wanting, housing provided by the CMHA housing program or LMCH.

4. Diagnosed with a psychotic or major mood disorder.

5. Identified by the clinical team to require prompting/reminding to complete activities of daily living and self- assessments as indicated by a score between 70 and 20 on the Social and Occupational Functioning Assessment Scale [15].

6. Must be between the ages of 18-85 years old and able to provide informed consent.

For both phases, this represents an opportunity sample as enrollment entirely depended on whether the participant is currently residing in the hospital or in the community. All participants have provided capable informed consent.

\section{Procedure}

The initial hospital prototype phase includes two hospital apartments located in two psychiatric inpatient facilities. Upon consenting to participate in the study, selected equipment was verified and approved by their HCP. The equipment was then delivered to the participant by the research coordinator and the research team's programmer who then sets up the devices and provides training to the participant. Training for usage of the CHR is provided by the research coordinator with the HCP in a one-to-one session. The participant completes their first interview upon discharge from the hospital prototype apartment. The participants complete a second interview at 6 months postdischarge. 
The community phase is an expansion of the hospital prototype phase but participants are not crossed over, meaning that those who are discharged from the hospital prototype apartments do not enter the community phase. Upon enrollment into the study, participants for this phase of the project complete a baseline interview consisting of questionnaires pertaining to demographic data, health, housing, community integration and service utilization. Follow-up interviews are then conducted at 6 months and 12 months.

Focus groups are being provided to HCPs for both phases at the study's end allowing them to provide their observations and thoughts on the use of smart technology for the participants. A focus group at the study's end for participants will only be made available for participants in the community phase.

\section{Instruments}

In both phases of the study, the participants complete semi-structured interviews that include the following assessment tools: Community Integration Questionnaire Revised (CIQ-R), Short-Form 36, EQ5D, the Housing History survey, the Health, Social and Justice Service Utilization (HSJSU) questionnaire, and the Perception of Smart Technology Questionnaire, a researcher-developed questionnaire that inquired about participants' attitudes and opinions of the equipment provided to them. Demographic data is also collected during these interviews.

Health data from the health monitoring devices include blood pressure, weight, blood glucose levels and heart rate. This data is backed up to the LIDB and made available to their HCPs for monitoring and tracking. Apps for these devices are preloaded onto the smartphones and tablets so that participants can also monitor their data. Participants could use the devices as and when they wished, or if directed by their HCP.

\section{Data Analysis}

Data is entered and stored on REDCap, a secure web-based database application by a research assistant. For quantitative analysis, the research team uses SPSS Statistics Software to generate descriptive statistics. It should be noted that data from the CHR's Qnaires have not, and will not, be analysed. The primary outcome of interest is the total score from the CIQ-R. Further quantitative analyses will investigate the housing history of the participant to assess housing stability, the health status of the participant, and experience with health, social and justice services to evaluate service usage.

Individual interviews are held with all participants enrolled in the study. Focus groups, as described by Krueger [16], are held with HCPs in both phases as well as participants in the community phase. Research assistants will conduct qualitative analyses by applying a thematic grouping of responses by identifying recurrent themes and opinions expressed by the participants and HCPs. Specifically, an ethnographic method of analysis will be used to observe the broader social and cultural contexts surrounding individual experiences as well as the impact on HCPs and how the intervention influenced their practice [17].

A standardised evaluation framework facilitated systematic effectiveness, economic, ethical and policy analysis of outcomes [18].

Effectiveness analyses will utilise the mixed-methods approach of this study by analysing the quantitative data from the instruments provided during the individual interviews to assess for any changes or improvements to the participants' health, 
service utilization and community integration. Common qualitative items from the focus groups will also assess the usage of the technologies and suggestions for improvement. By using a mixed methods approach, the research team is able to ascertain the participants' experiences with the technology as well as the effects on health, housing stability, and community integration.

The economic analyses will focus on the value for money aspect of the intervention as well as the costs and benefits. Specifically, the health costs of the intervention compared to usual care as well as the costs of hospitalizations, emergency room visits, outpatient visits and home care service.

Quantitative and qualitative findings related to views of fairness, benefits and social inclusion will be compared to ethical standards derived from welfare theory and accepted ethical principles of care providers (i.e. autonomy, beneficence and respect) as part of the ethical analyses.

Finally, policy analyses will address the implications that arise from the issues identified in the interviews such as access to and utilization of services, housing history, impact of severity of illness, and need for personal resources.

\section{Results}

Data collection and enrollment into the study is currently still ongoing. However, of all the participants approached so far, none have rejected to participate in the study and none have participated in the study without any equipment. All participants so far have met the study with great enthusiasm and eagerness. In terms of acceptability of the devices offered, the participants in the study so far have been positive about having the smart technologies in their home. When asked the question "How do you feel about having these smart technologies in your home/hospital?" on the Perception of Smart Technology questionnaire, preliminary analyses have revealed an average of 6.5 out of 7 demonstrating favorable attitudes towards having the technology.

Participants who have completed their interviews did not indicate any changes to their health after using the smart technologies they had selected. However, the ability to track health changes was seen as a benefit of the health monitoring devices. Number of steps measured by the activity tracker and weight was seen as useful. Furthermore, one participant highlighted that these devices aided them in making healthier choices by checking their weight two to three times per week and reaching their goals for walking. This could therefore reflect an uptake in healthier lifestyle choices as the devices provide an extra level of incentive and accountability. Although screen devices could not be used in the prototype apartment based in a forensic setting, one participant noted they would like to have a screen device in order to use apps for nutrition and fitness.

One of the concerns that arose from an interview with one participant was the issue of using small screens for those with visual impairments. Although the tablet used was 10.1 in. in size, the text on the screen was still too small to accommodate visual difficulties, even when the font size had been increased. The touch-screen monitor however was able to remedy this as the screen size was double that of the tablet and therefore the participant was able to clearly see their prompts and reminders. One 
suggestion was to use sounds instead of text thereby reducing the need to read prompts and reminders.

Future analyses will be able to provide more in-depth data and knowledge pertaining to changes in community integration, health and housing stability over the course of the study and after discharge from the hospital prototype apartments. We will also be able to perform more detailed analyses of the effects of the smart technology on the participants and to what degree they had an impact on their lives.

Part of this project was also to observe whether a smart technology system would be feasible and whether it could be used in a mental health care environment. This project has demonstrated that this system has been capable of funnelling various forms of data from a number of different sources. Key to mental health care treatment and recovery planning is the ability to tailor the intervention specifically to the participant. This was achieved as participants could pick and choose the devices specific to their care plans with no change or drop in performance from the system.

\section{Discussion}

Developing a reliable and secure system is a crucial step before community adoption of the intervention and must ensure the privacy of users and their data. This initial testing performed in the hospital prototype apartments allowed for further developments and refinements before going live in community-based environments. The intervention has undergone a number of enhancements from the time of its conception, mostly as a result of the fast-moving pace of technology but also the upgrades and additions to existing software and capabilities that meet the needs of the HCPs and participants.

To the researchers' knowledge, there are no other community-integrated, systemslevel research studies underway that leverages novel state-of-the-art smart technology systems in community homes or in transitional hospital apartments. This project will advance the current knowledge of smart homes for individuals with severe mental illness by combining innovative health platforms (LIDB and CHR) and push smart technology to the forefront of mental health care within the home. With the infrastructure now in place, our team of researchers, health technology experts and software programmers are now equipped to support individuals with mental illness living in the community. Furthermore, this research project will have universal design potential across a range of mental illnesses for future research studies and/or interventions where optimal facilitation of healthy lifestyles may require smart technology support.

In terms of usage, the participants expressed they were generally satisfied with their usage of the devices provided to them. It was noted that the smartphone and tablet may be too small for individuals with visual impairments but the touch-screen monitor was able to provide suitable coverage. Training on using the devices was mostly straightforward as the activity tracker simply required the user to wear the device on their wrist and the weigh scale only required the user to stand on it. Similarly, the blood pressure monitor was easy to use, with the user strapping the cuff to their wrist then pushing one button located on the monitor attached to the cuff.

It has been reported so far that the health monitoring devices also helped to encourage weight and steps monitoring, which may be indicative of added motivation 
for the participants to maintain healthy lifestyles. The devices therefore may have acted as an accountability tool, providing the participants with their measurements and providing valuable feedback through enabling them to track their health. Previous studies have linked frequent observation to weight loss using personal digital assistants (PDAs) and daily feedback messages [19]. Research has also revealed that level of depression has been found to be a prognostic indicator for chronic illness such as coronary heart disease [20] and individuals with schizophrenia or depression have also demonstrated higher rates of diabetes and cardiac disease compared to the general population [3]. Therefore the use of health monitoring devices for individuals with mental illness cannot be understated.

A key benefit of this intervention would be the impacts on housing and economics. This project has allowed for the testing of commercial devices that can be easily purchased in the community or online and installed in community homes as opposed to expensive clinical equipment. This could inform housing policy decision-makers to consider the use of smart technologies for future housing development planning and for individuals with severe mental illnesses returning to the community. It is realistic to consider that future clinicians may prescribe smart devices and personalised health care technologies to support treatment or recovery. As the cost of mental care across Canada has been estimated to cost billions of dollars, this intervention may propose a more cost-effective alternative. At present, the cost of fitting an apartment with the devices plus CHR licensing and setup, and phone plan bills is less than $\$ 20,000$ per year. St. Joseph's Hospital in London, ON, has estimated the mental health per diem cost as $\$ 454$ per day ( $\$ 165,710$ per year) with a total of 10,699 patient days per year; which amounts to a net cost of $\$ 4,857,346$ per year with the most prolonged stays being those with difficulty finding housing due to functional issues. Further costs can be saved by reducing travelling and improving efficiency in managing caseloads through greater monitoring and time-saving communication for HCPs [21].

\section{Challenges}

Many smart devices do not meet the security or privacy requirements of such a system or the compatibility requirements to connect with the other selected smart devices. For example, six different activity trackers were reviewed before one met the security and compatibility criteria. Finding a Cloud where it's Application Programming Interface (API) would grant the research team easy access to the data to be exported to the LIDB also proved to be challenging.

A challenge for this project was encouraging HCPs to adopt this technological approach. Although a number of HCPs embraced the technology and efficiency of the software available, others were skeptical. Due to the personal nature of the data being collected, some were concerned about the participants' privacy. However, concerns were allayed during the training sessions with HCPs as monthly meetings had been held between the hospital's IT and Privacy departments and the research team prior to the study's commencement. Some HCPs in the forensic setting felt more comfortable seeing the participant in person due to the close proximity between the prototype apartment and the HCP's office instead of viewing the CHR or the health data exported to the LIDB. 
As one of the inpatient psychiatric facilities was a forensic mental health institution, a number of security protocols needed to be addressed. Internet usage specifically was prohibited due to the nature of the institution and the ease of access for harmful, dangerous or offensive materials on the internet. This therefore included the prohibition of screen devices that can connect to the internet. Further, devices with the ability to take photos or videos were also prohibited. The touch-screen monitor was acceptable as it could not be used for web-browsing and was programmed only to receive reminders as well as send acknowledgements back to the LIDB. However, this meant that the functions of a smartphone or tablet were not able to be replicated. The health monitoring devices were allowed as these did not allow for internet web-browsing.

\section{Conclusions}

Based on the preliminary findings of the current studies, the use of smart technology offers an alternative to traditional mental health care plans by allowing for enhanced connectivity and greater access to resources. The system described in this article is an efficient and reliable form of data management that can allow for tracking and monitoring of physical and mental health data. This intervention could enable mental health care strategies and inform policy decision-makers to adopt more smart technologies into care and treatment plans for individuals with severe mental illness. In addition, if implemented it can be cost-effective for individuals with mental illness living in the community and the lack of access to non-emergency health care services, the latter of which leads to an increase in emergency department usage and therefore funding. In providing a quality-assured smart technology system, individuals experiencing severe mental illness can gain access to mental health services that they may not have been able to access previously. Critical to this was the initial prototype apartment testing to ensure the intervention met the standards of those living in the community and their care teams. Full acceptability of smart technology within mental health care could support quality of care and improve integration for individuals living in the community. This can happen by combining mental health care legislation with public awareness to allay fears of personally identifiable data falling outside of the individual's circle of care, and assuring patients/tenants/HCPs that third parties will not have access to their information as the system is capable of managing electronic medical records.

Acknowledgments. We would like to acknowledge St. Joseph's Health Care, the Canadian Mental Health Association and London Middlesex Community Housing for facilitating the research environment. We would like to thank the participants and HCPs for their voluntary participation. Also, we appreciate the commitment of the research assistants for data collection and auditing to make sure the quality of research is assured. Finally, we would also like to acknowledge the two granting agencies - Canadian Mortgage \& Housing Corporation, and Canadian Institutes for Health Research - for making the funding available.

Conflicts of Interest. The authors declare no conflict of interest. 


\section{References}

1. Mental Health Commission of Canada: Making the Case for Investing in Mental Health in Canada. Mental Health Commission of Canada, Ottawa (2013)

2. Poirier, S., et al.: Schizophrenia patients with polydipsia and water intoxication are characterized by greater severity of psychotic illness and a more frequent history of alcohol abuse. Schizophr. Res. 118, 285-291 (2010)

3. De Hert, M., et al.: Physical illness in patients with severe mental disorders. I. Prevalence, impact of medications and disparities in health care. World Psychiatry 10, 52-77 (2011)

4. Dyck, K.G., Hardy, C.: Enhancing access to psychologically informed mental health services in rural and northern communities. Can. Psychol. 54, 30-37 (2013)

5. Walker, E.R., McGee, R.E., Druss, B.G.: Mortality in mental disorders and global disease burden implications: a systematic review and meta-analysis. JAMA Psychiatry 72, 334-341 (2015)

6. Ho, A., Quick, O.: Leaving patients to their own devices? Smart technology, safety, and therapeutic relationships. BMC Med. Ethics 19, 18 (2018)

7. Forchuk, C., Csiernik, R., Jensen, E. (eds.): Homelessness, Housing and Mental Health. Canadian Scholars' Press, Toronto (2011)

8. Corring, D., Campbell, R., Rudnick, A.: A smart apartment for psychiatric inpatients. Psychiatr. Serv. 63, 508 (2012)

9. Corring, D., Meier, A., Rudnick, A., Forchuk, C.: Using mobile technology to promote independence: an innovation in psychiatric rehabilitation - a feasibility study. Res. Insights 12, 2-6 (2015)

10. Forchuk, C., et al.: TELEPROM-G: a study evaluating access and care delivery of telehealth services among community-based seniors. In: Future Technologies Conference: Proceedings of Future Technologies Conference, pp. 1346-1348. IEEE, San Francisco (2016)

11. Forchuk, C., et al.: Mental health engagement network (MHEN). Int. J. Adv. Life Sci. 5, 110 (2013)

12. Forchuk, C., et al.: Mental health engagement network: an analysis of outcomes following a mobile and web-based intervention. J. Technol. Soc. 11, 1-10 (2015)

13. Garafalo, J., Nathan-Roberts, D.: Assessment of the impacts from the addition of novel assistive technologies in mental health care. In: Proceedings of the Human Factors and Ergonomics Society 2016 Annual Meeting, vol. 60, pp. 1245-1248. Sage, Washington DC (2016)

14. Martin, S., Kelly, G., Kernohan, W.G., McCreight, B., Nugent, C.: Smart home technologies for health and social care support. Cochrane Database Syst. Rev. 4 (2008)

15. Goldman, H.H., Skodol, A.E., Lave, T.R.: Revising axis V for DSM-IV: a review of measures of social functioning. Am. J. Psychiatry 149, 1148-1156 (1992)

16. Krueger, R.A.: Focus Groups: A Practical Guide for Applied Research. Sage Publications, Thousand Oak (1994)

17. Leininger, M.M.: Ethnography and Ethnonursing: models and modes of qualitative analysis. In: Leininger, M.M. (ed.) Qualitative Research Methods in Nursing, pp. 33-72. Grune \& Stratton, Orlando (1985)

18. Forchuk, C., Rudnick, A., MacIntosh, J., Bukair, F., Hoch, J.S.: Evaluation framework for smart technology mental health interventions. In: Chang, C., Chiari, L., Cao, Y., Jin, H., Mokhtari, M., Aloulou, H. (eds.) ICOST 2016. LNCS, vol. 9677, pp. 203-210. Springer, Cham (2016). https://doi.org/10.1007/978-3-319-39601-9_18

19. Turk, M.W., et al.: Self-monitoring as a mediator of weight loss in the SMART randomized clinical trial. Int. J. Behav. Med. 20, 556-561 (2013) 
20. Nicholson, A., Kuper, H., Hemingway, H.: Depression as an aetiologic and prognostic factor in coronary heart disease: a meta-analysis of 6362 events among 146,358 participants in 54 observational studies. Eur. Heart J. 27, 2763-2774 (2006)

21. Wurster, A.E., Archer, N.: Smart home technology and the needs of the aging population in Southern Ontario. Working Paper No. 59. McMaster eBusiness Research Centre (2016)

Open Access This chapter is licensed under the terms of the Creative Commons Attribution 4.0 International License (http://creativecommons.org/licenses/by/4.0/), which permits use, sharing, adaptation, distribution and reproduction in any medium or format, as long as you give appropriate credit to the original author(s) and the source, provide a link to the Creative Commons license and indicate if changes were made.

The images or other third party material in this chapter are included in the chapter's Creative Commons license, unless indicated otherwise in a credit line to the material. If material is not included in the chapter's Creative Commons license and your intended use is not permitted by statutory regulation or exceeds the permitted use, you will need to obtain permission directly from the copyright holder. 